Article

\title{
WiSPH: A Wireless Sensor Network-Based Home Care Monitoring System
}

Pedro Magaña-Espinoza ${ }^{1}{ }^{*}$, Raúl Aquino-Santos ${ }^{1}$, Néstor Cárdenas-Benítez ${ }^{2}$, José Aguilar-Velasco $^{2}$, César Buenrostro-Segura ${ }^{2}$, Arthur Edwards-Block ${ }^{1}$ and Aldo Medina-Cass ${ }^{2}$

1 College of Telematics, University of Colima, Avenida Universidad 333, C.P. 28045 Colima, Col., Mexico; E-Mails: aquinor@ucol (R.A.-S.); arted@ucol.mx (A.E.-B.)

2 Department of Innovation and Technological Development, Siteldi Solutions S.A. de C.V., 111 Canario Street, C.P. 28017 Colima, Col., Mexico;

E-Mails: nestor.cardenas@ siteldisolutions.mx (N.C.-B.); jose.aguilar@siteldisolutions.mx (J.A.-V.); cesar.buenrostro@ corporativostr.com (C.B.-S.); aldo.medina@corporativostr.com (A.M.-C.)

* Author to whom correspondence should be addressed; E-Mail: pedro_magana@ucol.mx; Tel.: +52-312-316-1075.

Received: 19 March 2014; in revised form: 11 April 2014 / Accepted: 11 April 2014 /

Published: 22 April 2014

Abstract: This paper presents a system based on WSN technology capable of monitoring heart rate and the rate of motion of seniors within their homes. The system is capable of remotely alerting specialists, caretakers or family members via a smartphone of rapid physiological changes due to falls, tachycardia or bradycardia. This work was carried out using our workgroup's WiSe platform, which we previously developed for use in WSNs. The proposed WSN architecture is flexible, allowing for greater scalability to better allow event-based monitoring. The architecture also provides security mechanisms to assure that the monitored and/or stored data can only be accessed by authorized individuals or devices. The aforementioned characteristics provide the network versatility and solidity required for use in health applications.

Keywords: wireless sensor networks; WiSPH; home care monitoring systems; fall detection; e-Health 


\section{Introduction}

\subsection{Research Background and Motivation}

Globally, an aging population provides a good indicator of how health services have progressed in both developed and developing countries. However, to better provide these benefits, a series of challenges must first be met. One extremely important challenge is to train much needed healthcare professionals who specialize in providing care to seniors who often suffer from a variety of chronic diseases associated with aging, and design environments that incorporate wireless technologies and communications systems adapted to the needs of the geriatric community [1]. Projections show that between 2000 and 2050 the number of people above the age of 60 will increase from $11 \%$ to $22 \%$ worldwide, meaning that persons in this age group will number approximately 2 billion [2].

Aging presents a series of challenges for the entire world population, primarily because seniors slowly lose their ability to be self-sufficient due to chronic diseases, physical and/or mental disabilities, or the general frailty that characterizes the aging process [2]. Any of these conditions represent factors that limit the elderly or endanger their lives, even within the confines of their homes. Consequently, 24-hour-a-day monitoring of the elderly can improve attention provided for chronic or acute health concerns, accidents such as falls, as well as a series of other conditions that can detrimentally affect the elderly. For example, falls represent the second most common cause of death by accident among the aged, making persons over the age of 60 the most vulnerable population group. Additionally, non-fatal falls by the elderly can severely compromise quality of life and/or represent considerable medical expenditures (i.e., in Finland $\$ 3,611$ dollars per injury, in Australia $\$ 1,049$ dollars per injury) [3].

Providing remote healthcare monitoring and services presents a series of important challenges; therefore, it is important to generate remote monitoring strategies to provide primary healthcare services and mechanisms that allow seniors to receive long-term assistance. To better meet the needs of the aging population, research has significantly advanced both the theory and application of e-Health technologies; largely because their application can reduce costs generated by patient monitoring and provide a variety of advanced services [4]. Importantly, studies show that the elderly generally accept e-Health technologies and consider them beneficial [5].

\subsection{Research Objective}

The main objective of this research was to design, develop and implement a system (WiSPH) in conjunction with IEEE 802.15.4 to detect and alert trained professionals about persons falling to the ground, as well as event-based monitoring to report tachycardia and bradycardia [3]. The objectives of this work include:

1. developing a fall detection algorithm to handle data transmitted from an accelerometer;

2. generating an event-based, reliable and scalable network algorithm;

3. employing a reliable data encryption scheme (AES ) for use within the WSN;

4. creating a domestic WSN infrastructure which allows patients freedom of movement without losing communication; 
5. programming a mobile application and Web page for easy access to live monitoring and information access;

6. transmitting push notifications of abnormal events to be relayed to caretakers, doctors or family members.

\section{Related Work}

\subsection{E-Health Applications}

E-Health applications are gaining popularity and greater acceptance because of their versatility and reduction of care-taking costs. Nowadays diverse systems and health-centered applications are being developed, which according to their application can be categorized in: daily living activities, fall and movement detection, location tracking, medication intake and medical status monitoring [6]. Applications for daily living activities focus on monitoring the activities of individuals within a predetermined space. One important example of this type of project is AICO, which utilizes a sophisticated combined Bayesian network that includes the input from a series of environmental parameters that can create an approximation of the activities being carried out by an individual [7]. Another application of this kind is Caregiver's Assistant, which employs RFID cards and a database that includes 38,000 human activities and a fast inference mechanism that allows persons to identify the actions of others remotely within a given space [8].

Applications that focus on fall and movement detection focus on following user movements and detecting falls. One example of this kind of service is Smart HCN, which consists of a WSN that monitors the posture of the subject and images taken by cameras to alert a specialist if the subject has suffered an accident [9]. Another example of an application that focuses on fall and movement detection is presented in work done by [10], which uses an accelerometer located at head-height to transmit data that can detect a fall and send a notification to a mobile personal digital assistant.

Location tracking applications are based mainly on the principle of identifying the location of users and analyzing their behavior. One example of this kind of application is presented in work done by [11]. This system employs a WSN to obtain RSSI values, which, through an algorithm, can locate the location of users within their homes. ZUPS is also an application that centers on location tracking for aged and/or disabled people. This project uses a ZigBee network and ultrasound-positioning systems, which allows caregivers to not only locate individuals within a specified space, but to also provide assistance for persons moving from one place to another beyond the confines of their home [12].

Medication intake applications consist mainly in monitoring the intake of the patient's drugs. iCabiNET provides a solution that employs a smart medicine manager that can notify patients via SMS or audio alarms at home to remind patients about their medications, as well as dosages and times [13]. Another medication intake application by the name of iPackage consists of medication wrappers with RFID tags which can be detected by an RFID sensor at the moment of ingestion, allowing caregivers to remotely monitor whether or not a patient is adhering to instructions [14].

Finally, medical status monitoring collects clinical variables (i.e., heart rate, glucose monitoring, pulse, etc.), elaborates a current-state diagnosis of the patient and provides the information to caretakers. If any abnormality is detected, it can be immediately communicated to either family 
members or caretakers. AlarmNet is an application that monitors a series of physiological variables, storing the data and processing the information to detect any abnormalities. If an abnormality is found, it notifies a mobile assistant [15]. Baby Glove is an application that monitors vital signs of newborn babies, such as their body temperature. The data is gathered through the baby's romper and then transmitted to a WSN, which constantly supervises important physiological variables and notifies caregivers if there is reading beyond the programmed limits in real time [16].

Although there are many e-Health applications available today, this work focuses on developing a hybrid application that focuses on fall and movement detection and on medical status monitoring in a controlled environment, based on a WSN, to detect falls, tachycardia and bradycardia for the elderly population. To achieve this, this research focused on the use of an accelerometer and a heart rate sensor, connected to a previously developed mobile monitoring node to collect data and send it to the WSN Infrastructure, only when abnormal readings are detected.

\subsection{E-Health Platforms}

E-Health applications can have different classifications, depending on their specific objective (fall detection, activity monitoring, localization, etc.). Several authors $[17,18]$ have identified a series of requirements for healthcare applications that are based on wireless technologies, including:

1. Reliability: the transmission of precise data, which involves preventing the duplication of information, by implementing an efficient Quality of Service $(\mathrm{QoS})$ which insures a high Packet Delivery Ratio (PDR) and reduces the Packet Loss Rate (PLR).

2. Energetic efficiency: the development or use of an energy-saving algorithm or technology to reduce the consumption of energy in devices, because this helps extend the life span of the network.

3. Data routing: the use of an efficient communication protocol that provides scalability, failure tolerance and best possible route selection, among others.

4. Node mobility: the ability of free moving wireless nodes to move within an indoor area, always maintaining optimum connectivity.

5. Security: the use of efficient security mechanisms to protect the data and privacy of highly sensible patient information, including its robbery or corruption.

Taking into account the proposed requirements for the development of a robust e-Health, Table 1 shows a comparative among the developed systems and platforms. Considering the requirements an e-Health solution, and taking into account the characteristics already existing platforms, our workgroup selected WiSe [19] (primarily because of its low energy consumption) with the goal of developing a robust, reliable system that integrates the best characteristics of the platforms/systems depicted in Table 1. 
Table 1. E-Health Platform Comparison.

\begin{tabular}{|c|c|c|c|c|c|}
\hline $\begin{array}{c}\text { Platforms/ } \\
\text { Requirements }\end{array}$ & Code Blue [20] & MEDiSN [21] & MASN [22] & KNOWME [23] & LOBIN [24] \\
\hline $\begin{array}{l}\text { Reliability } \\
\text { Mechanism }\end{array}$ & $\begin{array}{l}\text { None } \\
\text { (Unreliable } \\
\text { Multicast) }\end{array}$ & $\begin{array}{c}\text { Two-Tier } \\
\text { Architecture with } \\
\text { Dedicated Wireless } \\
\text { Backbone and } \\
\text { Optimized Rate } \\
\text { Control Protocols }\end{array}$ & $\begin{array}{c}\text { Dynamic } \\
\text { Reliability } \\
\text { Adaptation } \\
\text { Scheme }\end{array}$ & $\begin{array}{l}\text { Multithreading } \\
\text { collector }\end{array}$ & $\begin{array}{c}\text { Packet Loss Rate } \\
\text { tolerance } 2 \%\end{array}$ \\
\hline $\begin{array}{l}\text { Scheme for } \\
\text { Energy } \\
\text { Efficiency }\end{array}$ & Not Provided & $\begin{array}{l}\text { Division of } \\
\text { functionality } \\
\text { between acquiring } \\
\text { and relaying data }\end{array}$ & $\begin{array}{c}\text { Energy-aware } \\
\text { cluster formation } \\
\text { using energy } \\
\text { level } \\
\text { determination of } \\
\text { sensor nodes }\end{array}$ & $\begin{array}{c}\text { A combination of } \\
\text { data buffering, } \\
\text { adaptive sensor } \\
\text { throttling, and } \\
\text { dynamic selection } \\
\text { of data transmission } \\
\text { methods }\end{array}$ & $\begin{array}{c}\text { Assumes the loss of a } \\
\text { small percentage of } \\
\text { packets and compensates } \\
\text { bandwidth restrictions of } \\
\text { the IEEE } 802.15 .4 \\
\text { technology with a careful } \\
\text { design of several } \\
\text { communication protocols }\end{array}$ \\
\hline $\begin{array}{c}\text { Routing } \\
\text { Methodology }\end{array}$ & $\begin{array}{l}\text { Multicast and } \\
\text { multi-hop } \\
\text { routing }\end{array}$ & $\begin{array}{l}\text { Many-to-one and } \\
\text { one-to-one } \\
\text { communication }\end{array}$ & $\begin{array}{c}\text { Intra-Cluster and } \\
\text { Inter-Cluster Data } \\
\text { Relay }\end{array}$ & None. Star topology & Dynamic Source Routing \\
\hline $\begin{array}{l}\text { Techniques } \\
\text { for Mobility } \\
\text { Support }\end{array}$ & $\begin{array}{c}\text { Periodic } \\
\text { Flooding for } \\
\text { Route } \\
\text { Discovery }\end{array}$ & $\begin{array}{c}\text { Physiological } \\
\text { monitors, } \\
\text { periodically select } \\
\text { the best relay point } \\
\text { to forward their data }\end{array}$ & $\begin{array}{c}\text { None. Does not } \\
\text { support real-time } \\
\text { data collection } \\
\text { under mobility } \\
\text { conditions }\end{array}$ & $\begin{array}{c}\text { Nokia N95 } \\
\text { capabilities on } \\
\text { LAN/MAN } \\
\text { (WiFi, 3G, EDGE) }\end{array}$ & $\begin{array}{c}\text { Wireless } \\
\text { Communications } \\
\text { Infrastructure Subsystem }\end{array}$ \\
\hline Data Security & Not Provided & $\begin{array}{c}\text { 128-bits AES } \\
\text { encryption }\end{array}$ & $\begin{array}{c}\text { Only the } \\
\text { source/destination } \\
\text { can decipher the } \\
\text { medical data } \\
\text { through } \\
\text { crypto-keys }\end{array}$ & $\begin{array}{l}\text { End-to-end } \\
\text { encryption }\end{array}$ & Not Specified \\
\hline $\begin{array}{l}\text { Supported } \\
\text { Application }\end{array}$ & $\begin{array}{c}\text { Medical Care } \\
\text { and Disaster } \\
\text { Response } \\
\end{array}$ & $\begin{array}{l}\text { Emergency } \\
\text { Detection }\end{array}$ & $\begin{array}{c}\text { Real-time } \\
\text { collection of ECG } \\
\text { Data }\end{array}$ & General monitoring & $\begin{array}{l}\text { Real-time collection And } \\
\text { Emergency Detection }\end{array}$ \\
\hline $\begin{array}{c}\text { Sensor } \\
\text { Scalability }\end{array}$ & Not Specified & $\begin{array}{l}\text { supports motes with } \\
\text { different sensor } \\
\text { suites }\end{array}$ & $\begin{array}{c}\text { Yes, it can } \\
\text { increase the } \\
\text { number of } \\
\text { sensors (Does not } \\
\text { Specify which) }\end{array}$ & Not Specified & Not Specified \\
\hline
\end{tabular}

\section{Proposal Architecture}

This section describes the proposed network architecture. Figure 1 illustrates the general system. The proposed system architecture consists of: (a) Mobile monitoring node; (b) WSN Infrastructure; (c) SINK; (d) Remote monitoring and alert interfaces. 


\subsection{Architecture}

The mobile monitoring node receives, processes and forwards the data pertaining to tachycardia, bradycardia and falls throughout the networks. The WSN Infrastructure consists of a group of nodes placed throughout the home. These nodes are capable of establishing a hierarchical network, receiving information from the mobile nodes and routing the information to a computer with reduced computational capabilities and an 802.15.4 radio. The SINK is a computational device that picks up and stores the information it receives from the WSN infrastructure, permitting either local or remote access to the data. Finally, the Remote Monitoring and Alert Interface is an application to serve mobile devices and a standard website, both of which can receive notifications of important events and consult the patient's history and other personal information.

Figure 1. The proposed system architecture.

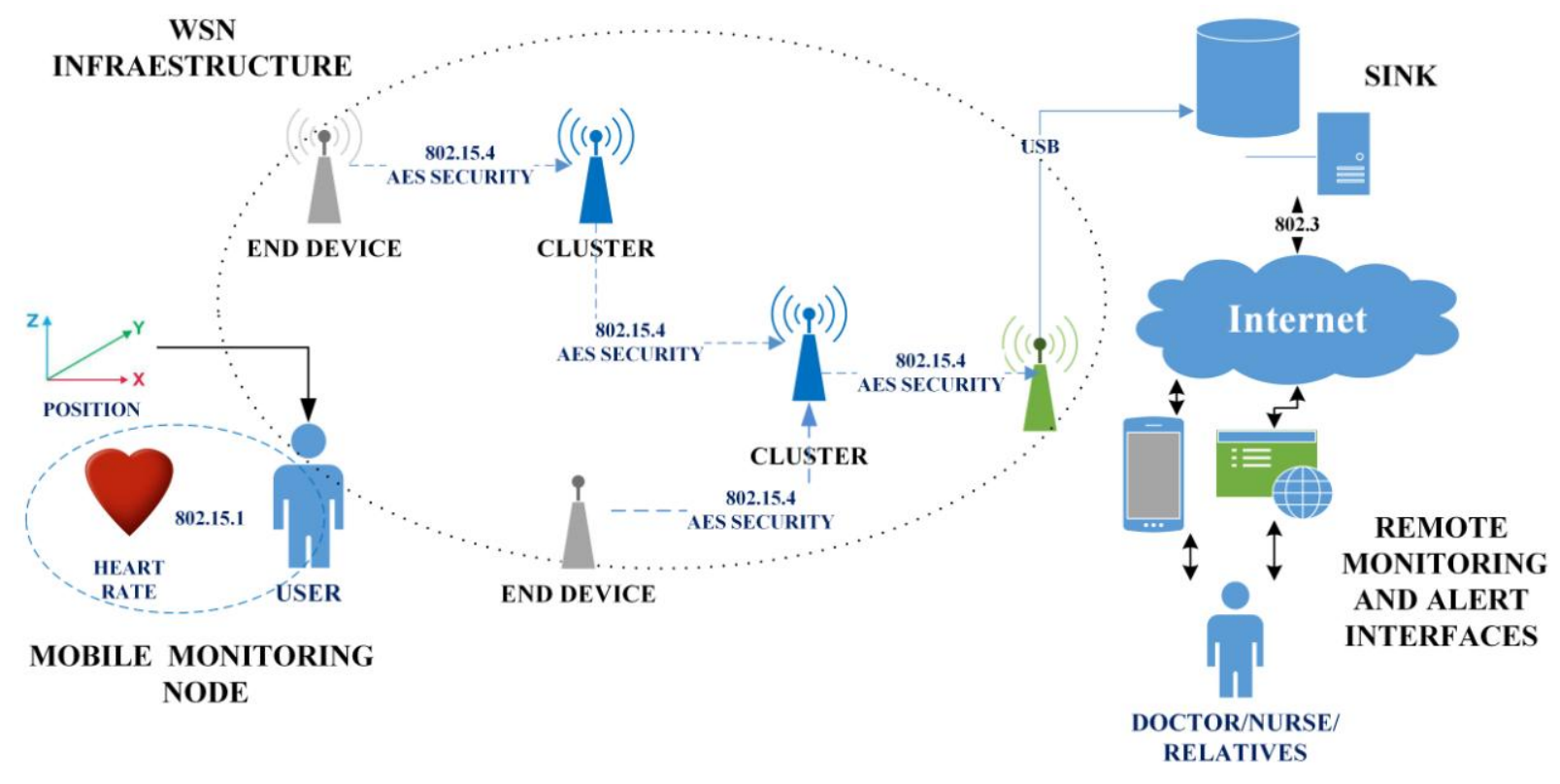

\subsection{Mobile Monitoring Node}

The mobile monitoring node consists of an LCP2148 ARM7 micro controller, a radio that is 802.15.4 compatible and a radio that is 802.15 .1 compatible, an energy module and a pair of sensors (accelerometer and heart rate sensor). The node is programmed to constantly monitor the values of the heart rate sensor and the accelerometer to detect abnormal values which could indicate an event that may compromise the user's safety. Figure 2 illustrates the Mobile Monitoring Node.

The triple axis accelerometer contained in the mobile device is used to detect rapid accelerations. These sensors are normally fabricated with small margins of error, which can be compensated for by means of software [25]. To calibrate each axis of the accelerometer, it is necessary to apply a different compensation value (offset) for each axis, $\left(a_{x}=1 \mathrm{~g}, a_{y}=0 \mathrm{~g}, a_{z}=0 \mathrm{~g}\right),\left(a_{x}=0 \mathrm{~g}, a_{y}=1 \mathrm{~g}, a_{z}=0 \mathrm{~g}\right)$, $\left(a_{x}=0 \mathrm{~g}, a_{y}=0 \mathrm{~g}, a_{z}=1 \mathrm{~g}\right)$. In order to deliver these samples, the micro-controller's Analogical to Digital Converter (ADC) is configured to $4.5 \mathrm{MHz}$ with a 10-bit resolution, which obtains the 3 axes at a $10 \mathrm{~ms}$ frequency and stores them. In order to calculate the offset, the $\mathrm{X}, \mathrm{Y}$ and $\mathrm{Z}$-axes were fixed at $0 \mathrm{~g}$. The value of the $\mathrm{ADC}$ in the $\mathrm{Y}$-axis when the acceleration is equal to $0 \mathrm{~g}$ is 505 ; the value of the 
$\mathrm{ADC}$ in the $\mathrm{X}$-axis when the value is $0 \mathrm{~g}$ is of 510 ; the value of the $\mathrm{ADC}$ in the $\mathrm{Z}$-axis with a $0 \mathrm{~g}$ acceleration is 515. Consequently, the three-axes of the accelerometer and the ADC values of each axis can be adjusted using the following formulas.

Figure 2. Block diagram of the Mobile Monitoring Node.

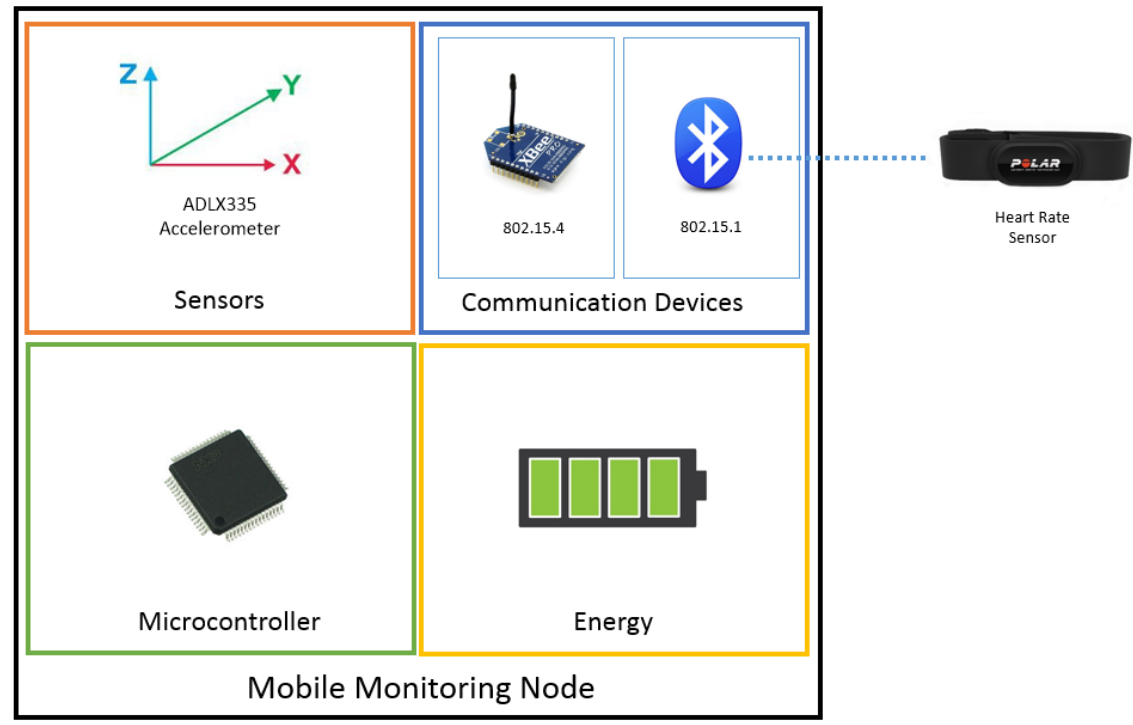

The calibration formula for the $\mathrm{Y}$-axis is:

$$
a_{y}=\frac{\left(\frac{A D C_{y} \times 3.3}{1024}\right)-1.63}{0.33}
$$

The calibration formula for the $\mathrm{X}$-axis is:

$$
a_{x}=\frac{\left(\frac{A D C_{x} \times 3.3}{1024}\right)-1.64}{0.33}
$$

The calibration formula for the $\mathrm{Z}$-axis is:

$$
a_{z}=\frac{\left(\frac{A D C_{z} \times 3.3}{1024}\right)-1.65}{0.33}
$$

The Signal Magnitude Vector (SMV) was calculated to detect a possible fall, which, according to [26], is $\mathrm{SMV}>1.8 \mathrm{~g}$.

The formula to calculate the SMV is:

$$
S M V=\sqrt{a_{x}^{2}+a_{y}^{2}+a_{z}^{2}}
$$

Once a possible fall has been detected, the PNR algorithm determines if a fall has actually occurred. The PNR algorithm is shown in Figure 3. The PNR algorithm activates when the SMV readings average $1 \mathrm{~g}$ to determine if the individual is on the floor and hurt due to an accident before it transmits a distress signal. Wang's algorithm, [10] which was used to validate falls, uses a sample rate of 60 SMV entries and compares each incoming entry with the 59 preceding samples. If the product of these 
samples is not above or the same as $0.13 \mathrm{~g}$, a value that is easily surpassed if the user stands up or walks normally, an alert will be sent to the network.

Figure 3. (a) Fall detection algorithm (part 1); (b) Fall detection algorithm (part 2).

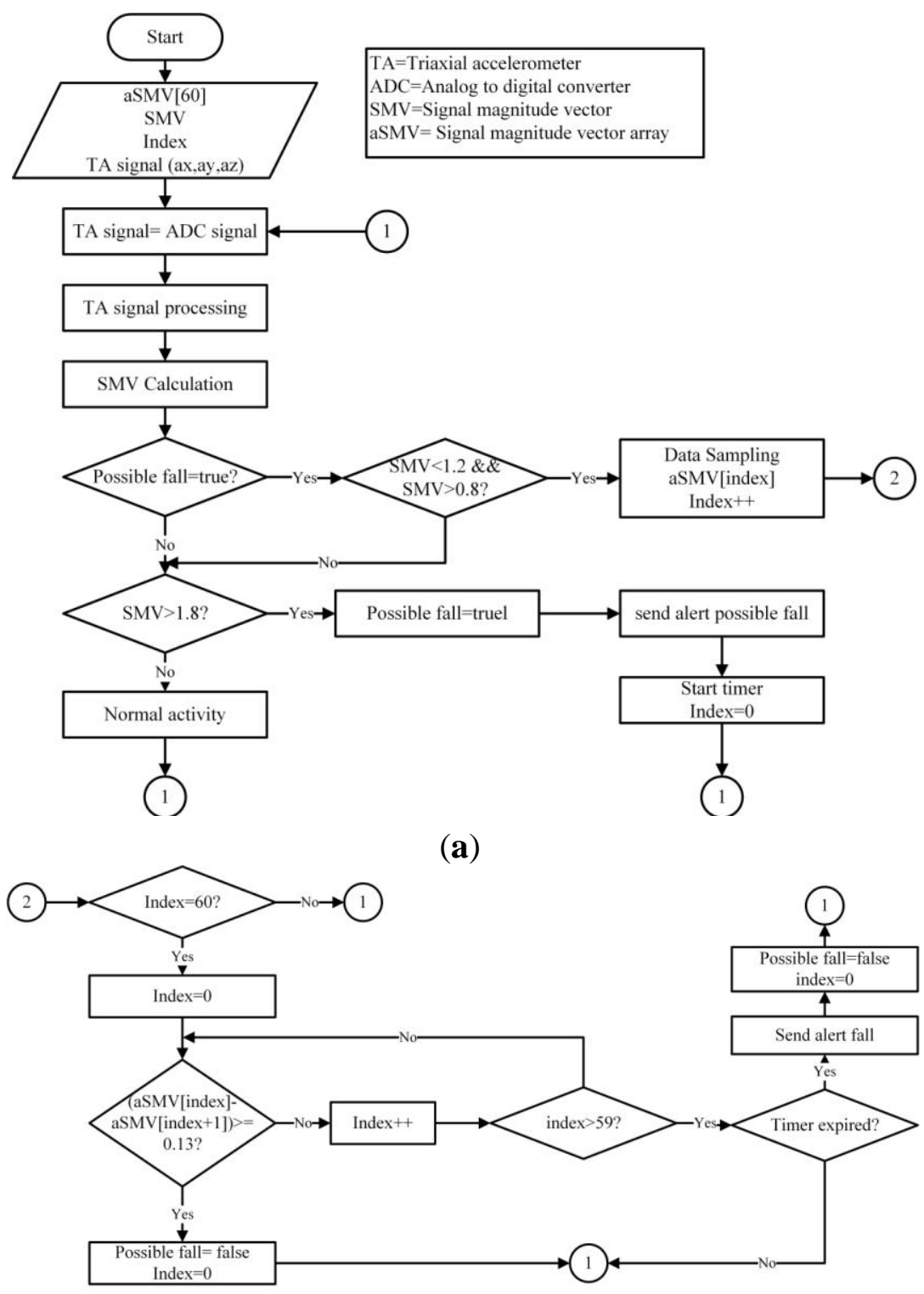

(b)

To detect abnormalities in the user's heart rate, a monitoring threshold was established based on beats per minute (BPM) measurements. One common heart rate abnormality is bradycardia, which is lower than 60 BPM for individuals at rest, although this condition may vary with age and daily habits of the individual. Older people, in particular, have bradycardia if their value drops below 50 BPM because the heart weakens and the heartbeat slows as persons age [27].

Another common heart beat abnormality is tachycardia. Adults with tachycardia have over 100 BPM [28]. As with bradycardia, a series of habits and age influence the values; however, these factors do not require in-depth study. Based on other research, the acceptable limits for tachycardia and bradycardia were set at 100 BPM and 60 BPM, respectively. Any values above or below the established limits would activate the alarm. 


\subsection{WSN Infrastructure}

The network used for the detection and transmission of data was developed using WiSe nodes; therefore, the nodes have a low energy consumption rate [19]. Low energy consumption is a very important consideration because the network life, integrity and security for health applications requires exceptional reliability [17].For these reasons, the WSN infrastructure employs a 128-bit AES encryption algorithm to protect network integrity and security, ensuring that the information is not intercepted by nodes that do not belong to the network [29].

A hybrid routing protocol that is dynamic, scalable, destination-centered, with a hierarchical structure was developed for the network. The system is based on IEEE 802.15.4, which contemplates the use of mobile nodes that form the network infrastructure. The infrastructure nodes handle the Received Signal Strength Indication (RSSI) to proactively create the network and can serve as: Cluster Head $(\mathrm{CH})$, End Devices (ED) and SINK. On the other hand, the mobile node only sends data when an event occurs (either and increased or decreased heart rate, or a fall). Therefore, the information is sent to a single network node that immediately reacts to the alert and finds the event destination. To cover this and other functions, the proposed routing algorithm uses a series of network packets which are described in Table 2.

Table 2. Packets generated by the routing protocol.

\begin{tabular}{ccl}
\hline $\begin{array}{c}\text { Type of Packet } \\
\text { HELLO }\end{array}$ & Length (Bytes) & \multicolumn{1}{c}{ Description } \\
\hline HACK & 11 & The packet code is $0 \times 48$. The HELLO packet discovers the network. \\
\hline TOKEN & 11 & $\begin{array}{l}\text { The packet code is } 0 \times 58 . \text { The HACK packet confirms reception of } \\
\text { the HELLO packet. }\end{array}$ \\
\hline TACK & $\begin{array}{l}\text { The packet code is } 0 \times 54 \text {. The TOKEN packet explores the network } \\
\text { before sending a DATA packet. }\end{array}$ \\
\hline DATA & $\begin{array}{l}\text { The packet code is } 0 \times 4 \text { B. The TACK packet confirms that the } \\
\text { TOKEN packet was received. }\end{array}$ \\
\hline & $\begin{array}{l}\text { The packet's code is } 0 \times 44 \text {. The DATA packet contains data about } \\
\text { the information's origin, as well as the patient's heart rate, the event } \\
\text { code, the number of hops and the packet counter. DATA packets, } \\
\text { however, can be adapted to carry even more information. }\end{array}$ \\
\hline
\end{tabular}

To create the network's structure, the nodes begin in an undefined state, except for the SINK. Each node periodically sends HELLO packets to localize any possible neighboring nodes. The HELLO packets are then broadcast and received by all of the immediate neighbors and is not retransmitted, this to prevent network flooding. Once the undefined nodes receive an answer (HACK packet) from a defined structure node (CH, ED or SINK), it will change its status (ED) and will stop transmitting the HELLO packets; then it will select the best link based on the Received Signal Strength Indication (RSSI). The functions of the nodes (ED) are to send DATA packets from a mobile node to its respective $(\mathrm{CH})$ and reply to the HELLO packets received from undefined nodes. When a node (ED) is connected to another infrastructure node, it creates and stores its new neighbor in a routing table; afterwards, it assumes the role $(\mathrm{CH})$. The $(\mathrm{CH})$ then routes the information received from the (ED and mobile nodes), maintaining the network nodes connected and replying to HELLO packets to the 
undefined infrastructure nodes. On the other hand, mobile nodes are already defined before joining the network. The primary purpose of a mobile node is to monitor user variables, discover the destination of the information monitored for each event and send DATA packets to the network.

The routing of DATA packets is carried out by a hybrid mechanism. A mobile node must first reactively identify its destination by broadcasting a TOKEN packet. Only upon receiving a (TACK) unicast reply from a network node will the mobile node send a return DATA package. Importantly, the DATA routing in infrastructure nodes is proactive because each node knows its route by default because the information is kept and actualized in its routing table. Figure 4 illustrates the proposed routing protocol for WSN.

Figure 4. (a) Routing Algorithm (part 1); (b) Routing Algorithm (part 2).

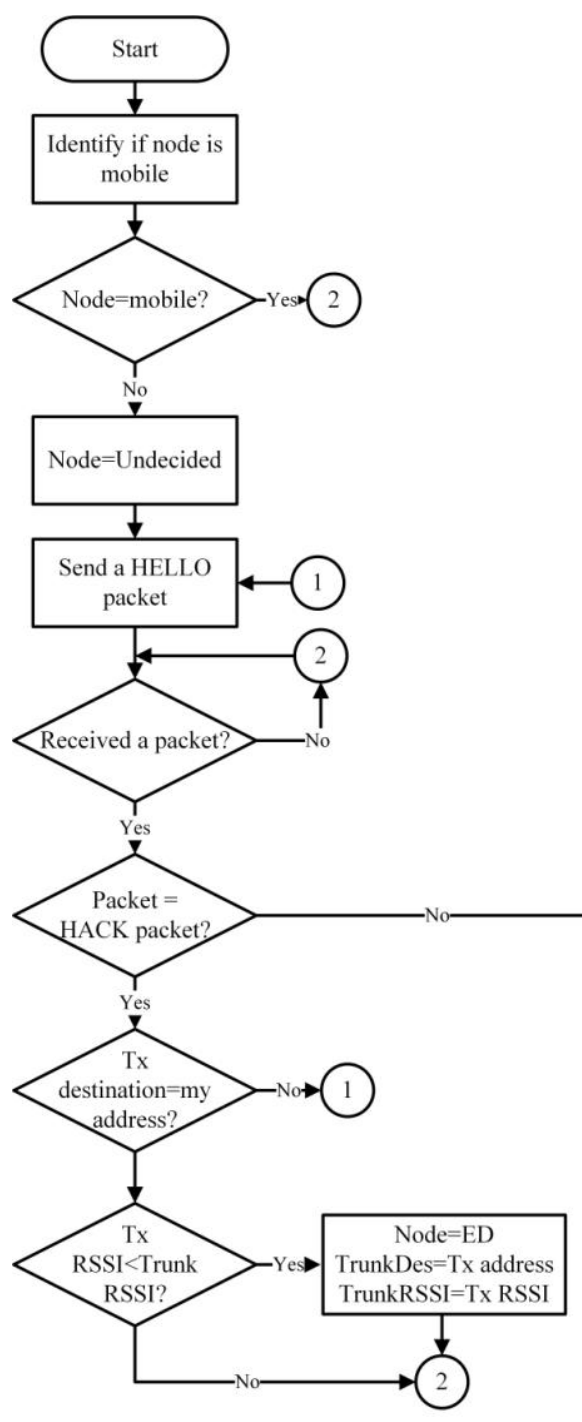

\begin{tabular}{l}
\hline RSSI = Received signal strength indication \\
HACK = Hello acknowledge packet \\
TACK = Token acknowledge packet \\
TrunkDes = Trunk destination \\
TemDest = Temporary destination \\
CH = Cluser Head \\
ED = End Device
\end{tabular}
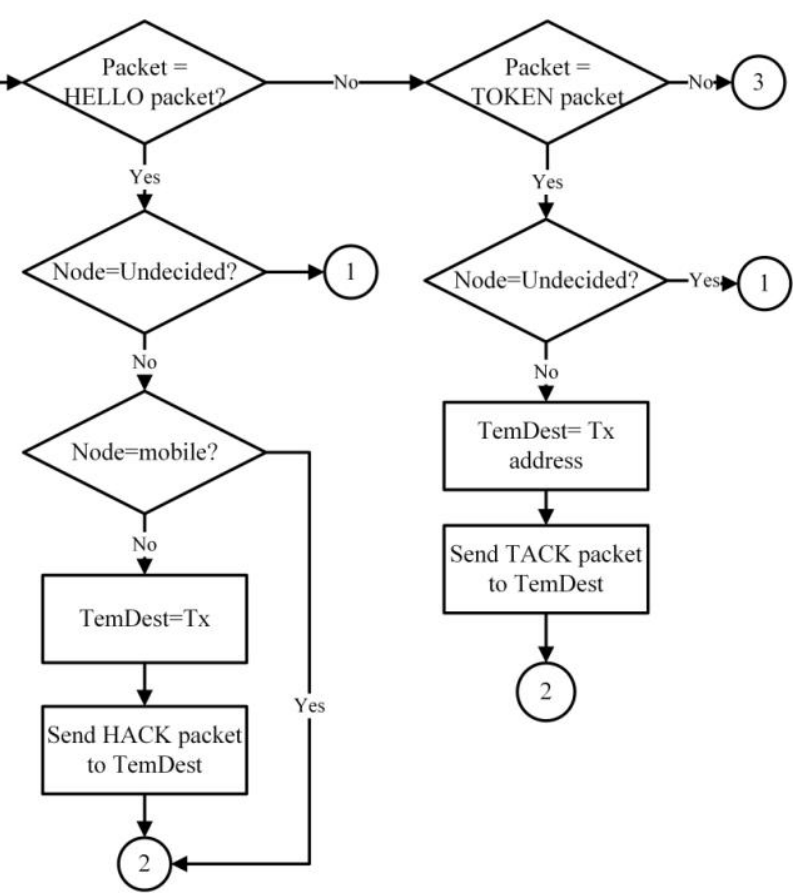

(a) 
Figure 4. Cont.

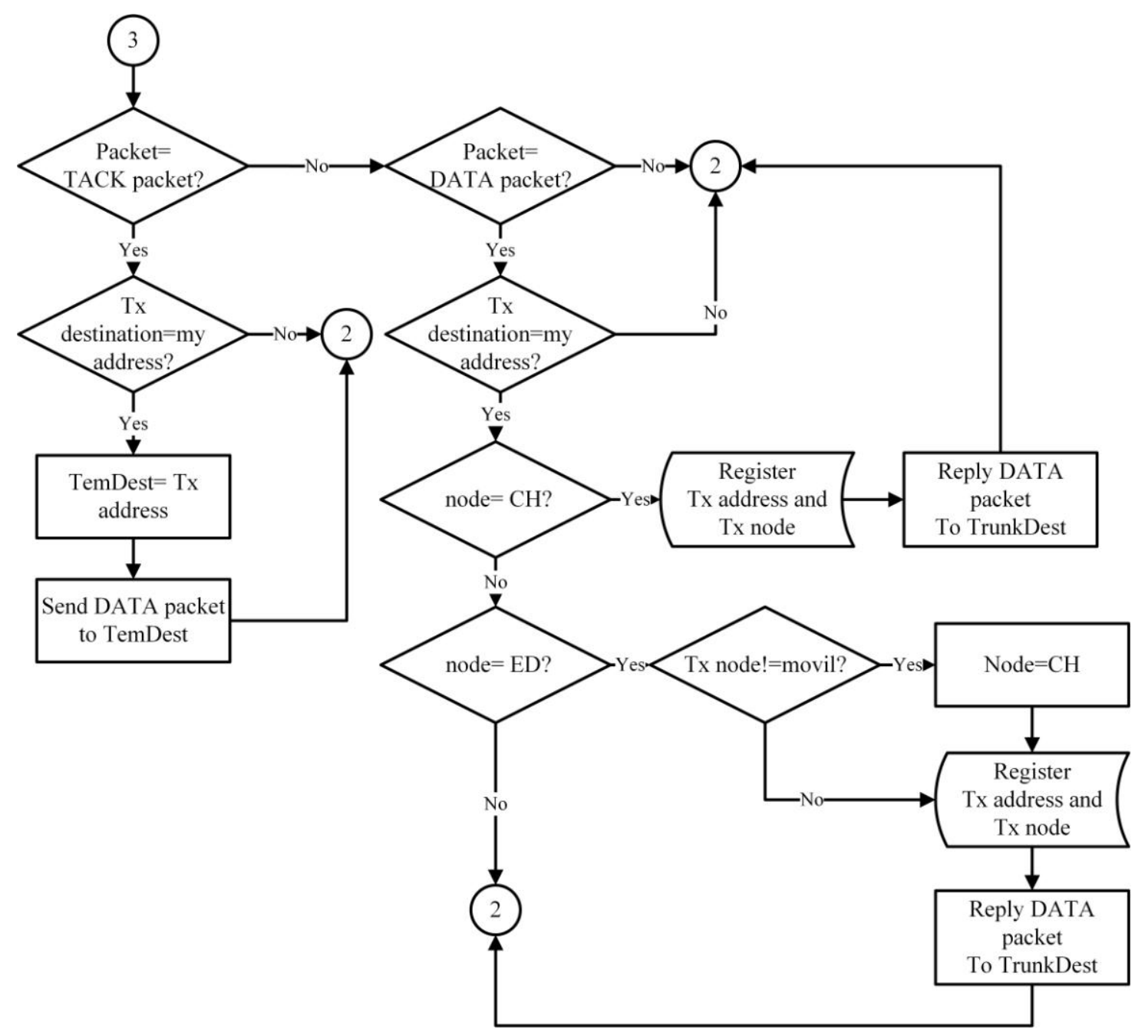

(b)

\subsection{SINK}

The SINK is comprised of electronic devices that have a greater processing capacity than the rest of the devices of the WSN Infrastructure, because it is where all the information compiled by the WSN is sent [30]. The SINK not only collects data, but communicates it through the Internet to the application that controls the database. The behavior of the data collector is shown in Figure 5. The behavior of the SINK is completely reactive as it waits for the arrival of any packet to be processed. When a new package is received, the type of packet must be analyzed. If it is a HELLO packet, the SINK creates a reply HACK packet, which contains information about the packet type, its origin (SINK ID), its destination (obtained from the transmitting node) and its role (role of the SINK). If the SINK receives a TOKEN packet, it sends a TACK packet to the transmitting node. If the SINK receives a DATA packet, it analyzes and classifies the packet content in order to store it in its database. 
Figure 5. SINK processing algorithm.

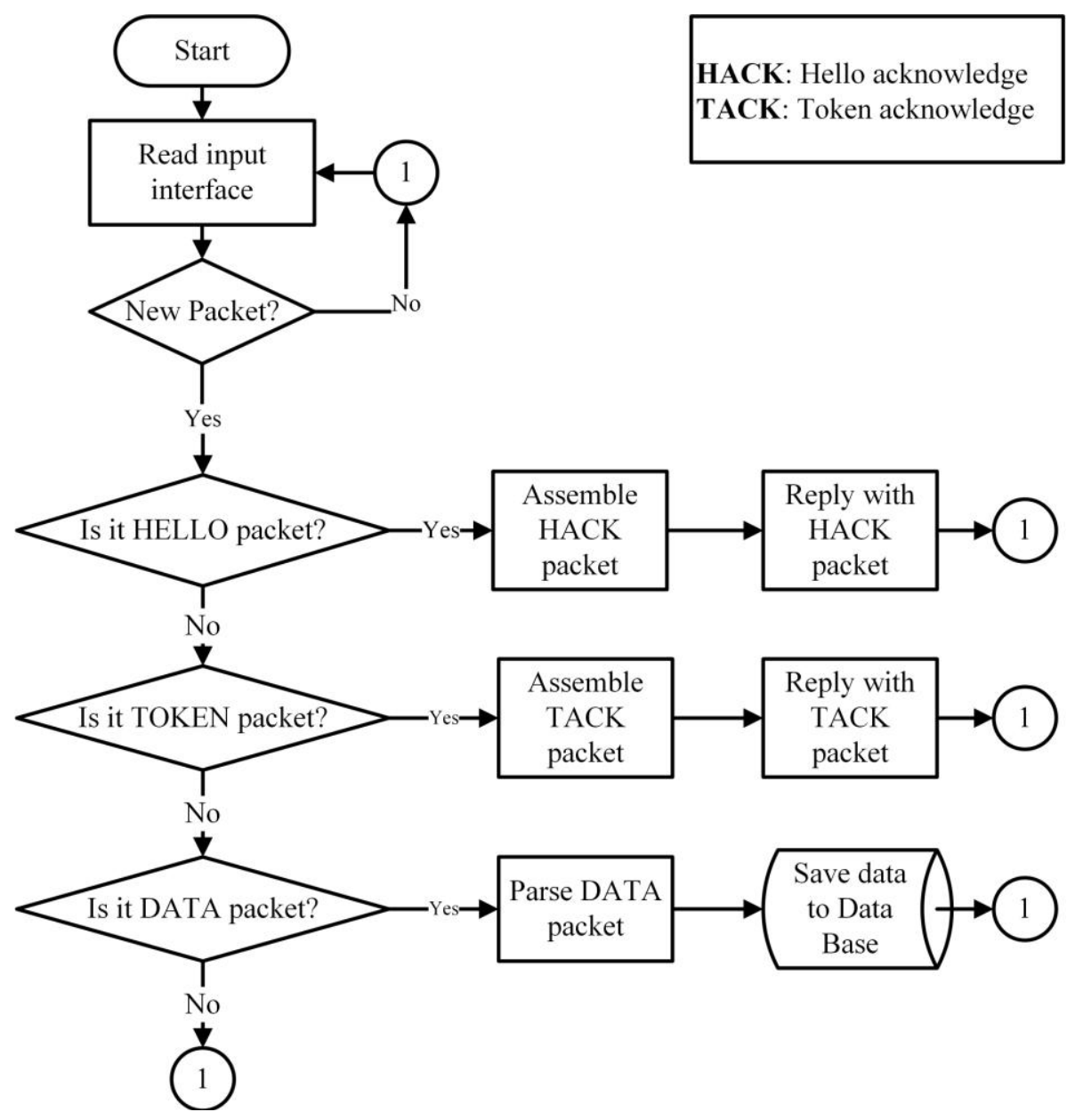

\subsection{Remote Monitoring and Alert Interface}

The web monitoring system consists of a front-end application which continually analyses every new entry sent to the database server. The system, in real time, can show a user's heart rate, possible and real falls, and can interpret the information and send notifications to caretakers in case of emergency. Figure 6 illustrates the interface functions.

The monitoring system functions reactively because it must first analyze the status of the database before it determines if the entry it is receiving is new. If the system does not detect any changes, an inactivity counter initializes and will compare the counter's reported values with previously set limits for inactivity. If the inactivity counter reports data that are below the established limit, it reinitializes. Importantly, if the inactivity counter reports are equal to the already established values, the system sends an alert message to the user to report a possible network error, because the system is not receiving new entries. In this way, the user can more easily determine if any problem exists. If the entry is new, the counter will automatically reset the counter and identify all the information to be displayed on the mobile or web interface.

Besides the alerts mentioned previously, the system also sends alerts relative to situations that may place the user's physical integrity, making it possible to notify those who are concerned for the patient's wellbeing about a potential fall or a bradycardia or tachycardia event. The alerts are generated upon analyzing any new entries received and recorded in the database. After receiving a new entry, the 
application analyzes it and validates the type of registry (fall or heart rate). Upon determining the type of registry, the alert counter resets, activating the alert mode and displaying the existing alert on the mobile device or Web page. After resetting, if there are no new entries registered in the database, the alert counter increases. Once the counter increases, the application compares the alert counter with the pre-established counter limits. If the entries surpass the alert counter limits, the alert mode is deactivated. This is an important safety measure as it prevents repeated and unnecessary alerts, or prolonged periods of system alerts.

Figure 6. Web interface and alerts flow chart.

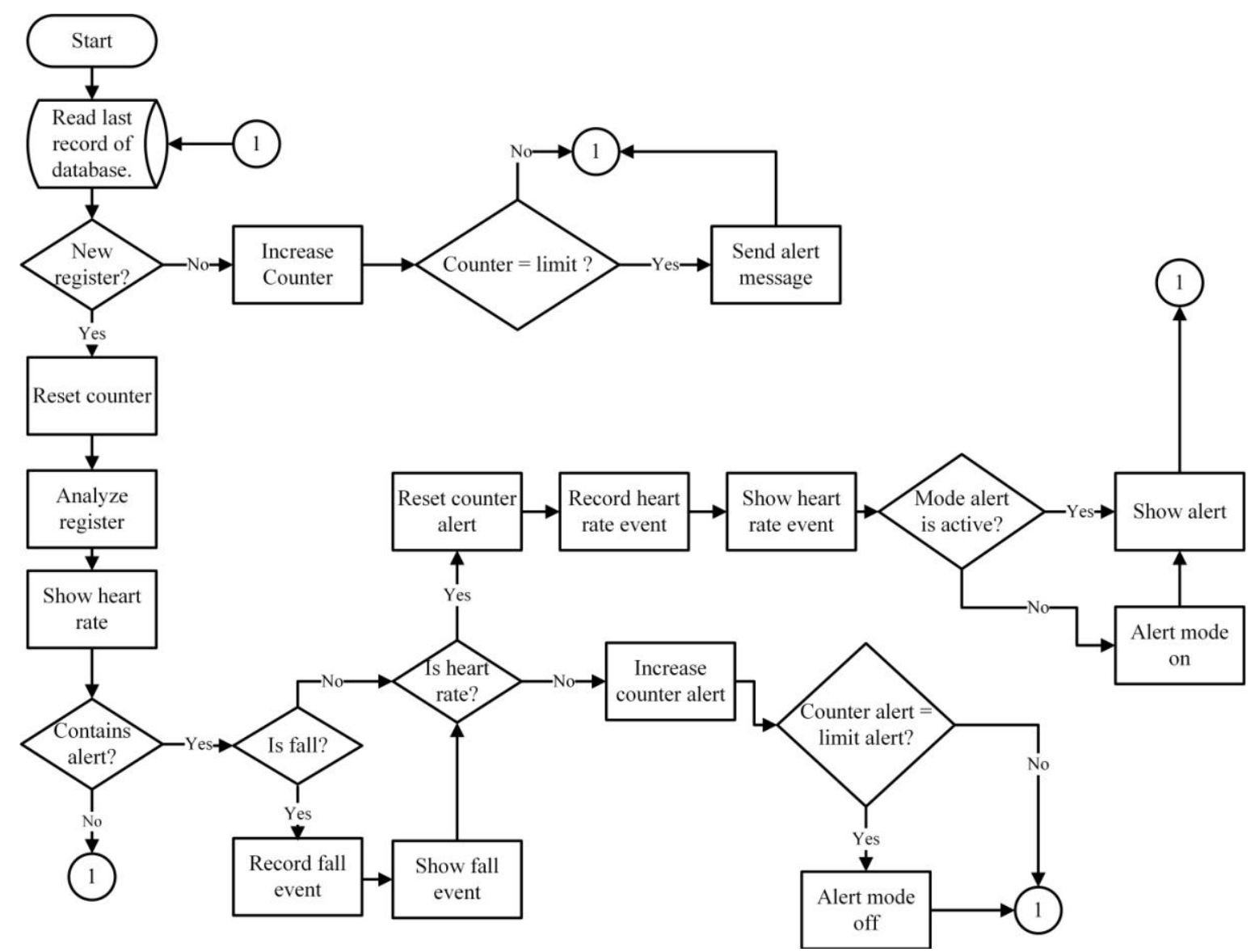

\section{Proof of Concept}

As a proof of concept for the proposed architecture, five WiSe nodes and one Mobile Node were deployed in a $14 \mathrm{~m} \times 20 \mathrm{~m}$ home, in order to mount a WSN infrastructure and ensure coverage throughout the entire home. A 128-bit AES encrypting mechanism was also used to secure all data transmitted throughout the network.

A total of 40 falls were carried out to validate the fall detection algorithm. The 40 falls were equally represented by 10 falls backwards, 10 falls forward, 10 falls to each side (five falls to the left and five falls to the right) and 10 falls to the knees, as shown in Figure 7. 
Figure 7. Fall tests.

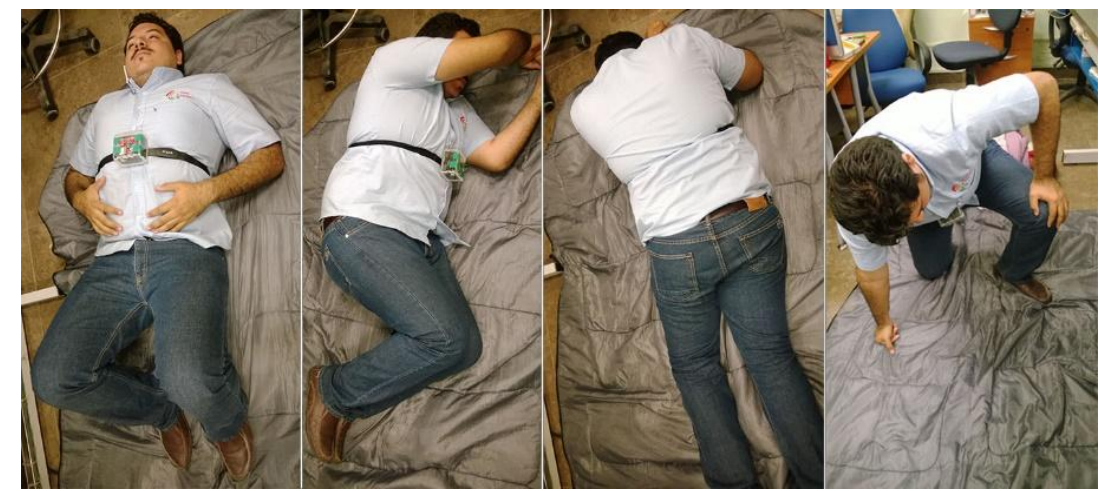

A mobile node was programed to send the events (DATA) to the WSN. The monitored data sent to the WSN was also sent to a second computer possessing graph processing software every $500 \mathrm{~ms}$ or upon occurrence of an event, which came first. This was done in order to validate the data actually received with the values of the proposed algorithm. To validate the established values for heart rates, 10 stress-related tests were conducted, all of which generated events that were sent throughout the network and stored in the monitoring and alert interface. Figure 8 presents the scenario in which the test was performed.

Figure 8. Proof of concept scenario.

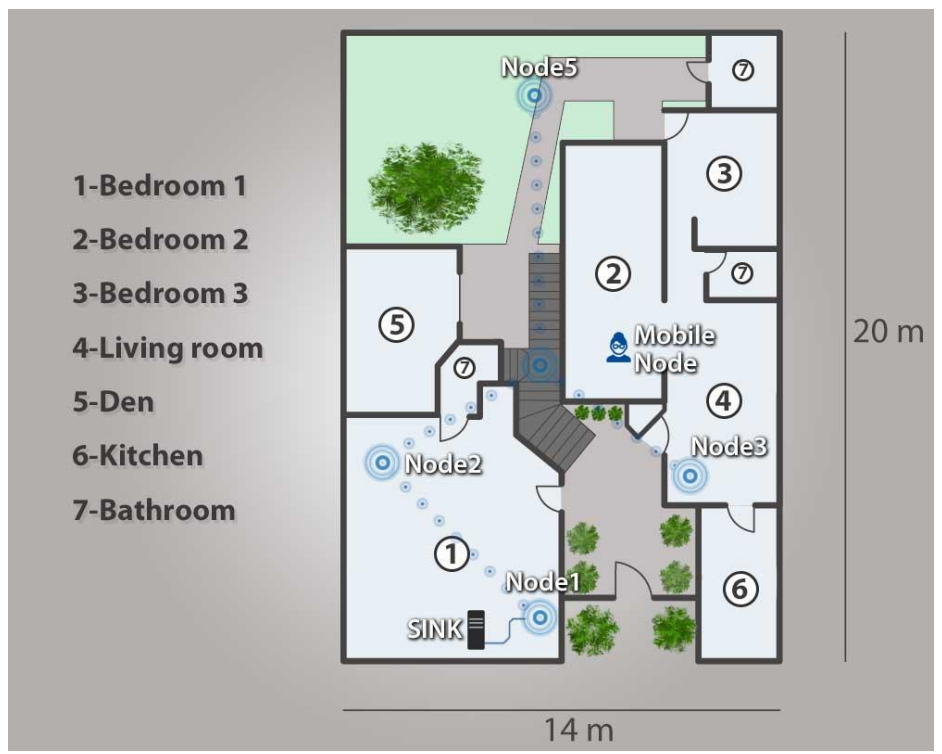

\section{Results}

This section provides the results of the proof of concept for the WiSPH system, which included:

(a) the PNR algorithm's ability to detect falls;

(b) the software's ability to detect abnormal heart rates;

(c) network metric results (RSSI, packet loss and hops). 


\subsection{Results of the PNR Algorithm for Fall Detection}

Data was transmitted throughout the network and to monitoring software after each event. However, the mobile node only relayed the values provided by the accelerometer to the monitoring software every $500 \mathrm{~ms}$. Figure 9 provides the results obtained from the 40 falls, where the four types of falls (the two types of side falls were categorized as one) captured by the monitoring software are shown. The monitoring software was reset for each kind of fall to assure more precise data. The tests reveal that the mobile node transmitted a total of 1,446 entries for the four types of falls to the monitoring software, which were then processed, analyzed and graphed. The black line in the graph reveals that the SMV remains lower than $1.8 \mathrm{~g}$ for normal everyday activities (walking, standing, standing up, sitting down, etc.). However, when the user falls, the line rises to levels above $1.8 \mathrm{~g}$, which causes the mobile to alert both the network and the monitoring software of a possible fall. After the simulated fall, subjects remained immobile on the ground, simulating being hurt. This was reported by the high peak of the black line in the graph. Significantly, when the black line does not exceed $0.13 \mathrm{~g}$ between entries for more than $30 \mathrm{~s}$ the mobile device sends a critical fall alert to both the network and the monitoring software.

In total, 56 possible falls and 41 critical falls were detected in the four tests, meaning that a total of 97 events were created. These events were then relayed across the network to the fall detection software. Only one false alarm occurred, which means that the PNR algorithm identified falls within the margin of error of $2.5 \%$. Table 3 shows the generated DATA packets by falls.

Figure 9. (a) Results from backwards falls; (b) Results from side falls; (c) Results from forward falls; (d) Results from knee falls.

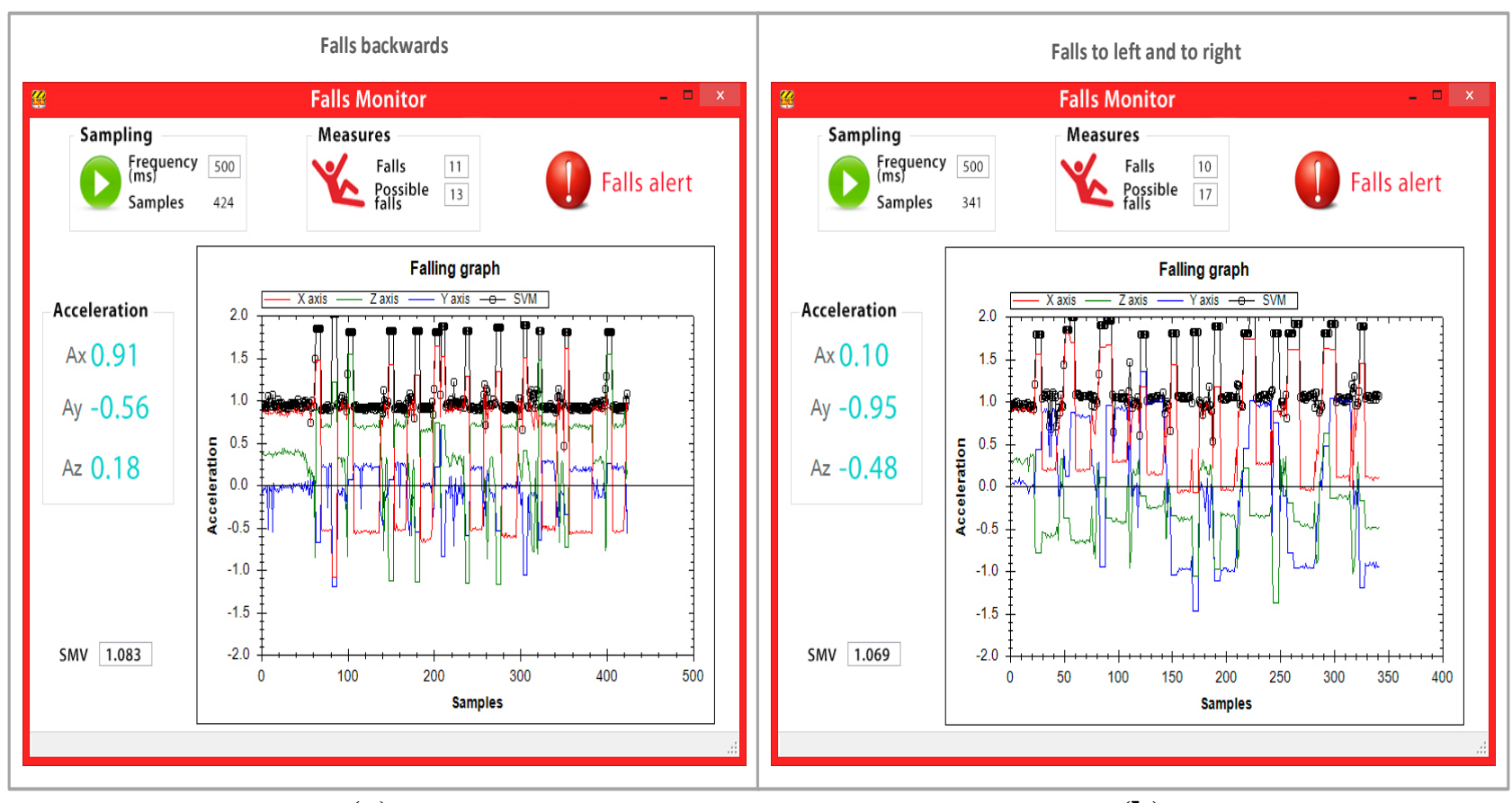

(a)

(b) 
Figure 9. Cont.

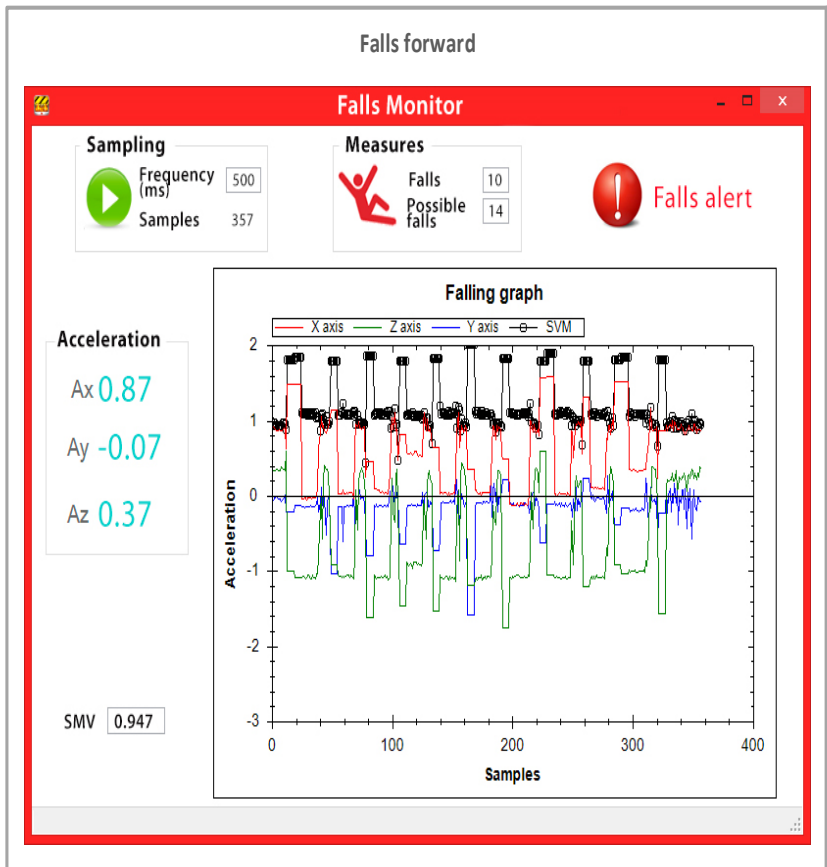

(c)

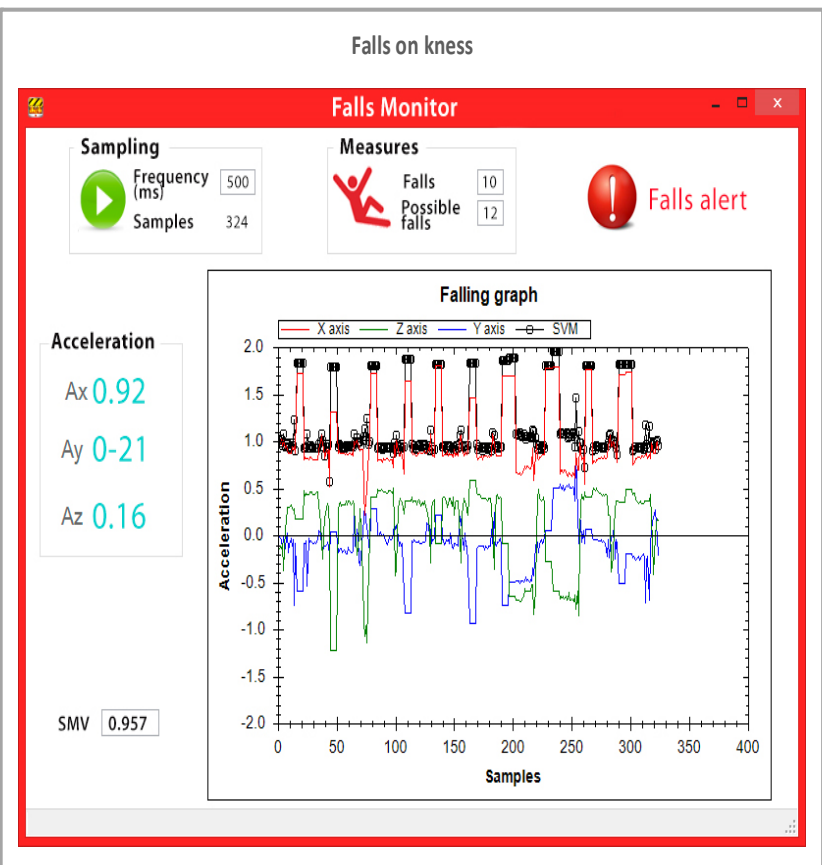

(d)

Table 3. Generated DATA packets from falls.

\begin{tabular}{ccccc}
\hline Type of Fall & $\begin{array}{c}\text { Total of } \\
\text { Programed Falls }\end{array}$ & $\begin{array}{c}\text { Total of } \\
\text { Possible Falls }\end{array}$ & $\begin{array}{c}\text { Total of } \\
\text { Detected Falls }\end{array}$ & $\begin{array}{c}\text { Total of } \\
\text { False Alarms }\end{array}$ \\
\hline Back & 10 & 13 & 11 & 1 \\
Side & 10 & 17 & 10 & 0 \\
Front & 10 & 14 & 10 & 0 \\
Knees & 10 & 12 & 10 & 0 \\
\hline
\end{tabular}

For each possible fall, alerts were generated and delivered to the caregivers by means of the application we developed for mobile devices, via push notifications. Figure 10 illustrates the mobile device interface.

Figure 10. Push notifications from the generated events.

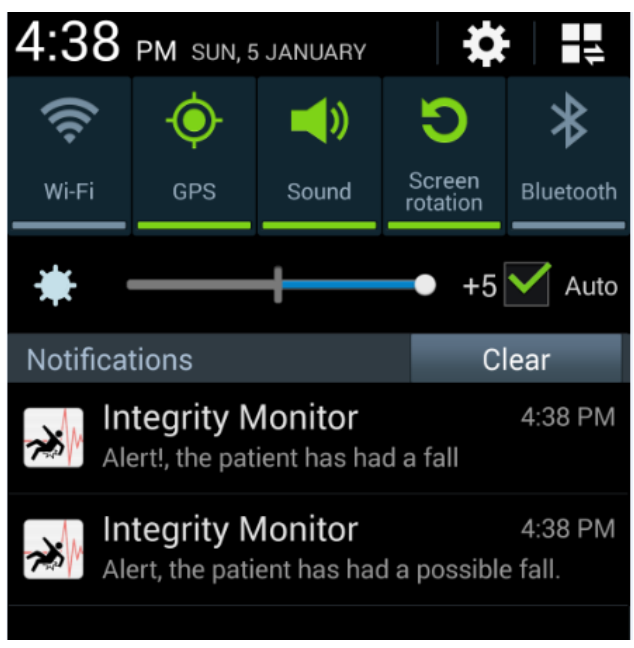




\subsection{Results from the Detection of Abnormalities in Heart Rate}

As was previously mentioned in Section 3.2, [27] and [28] established a monitoring threshold to detect heart rate abnormalities (bradycardia and tachycardia). They established a minimum value of $60 \mathrm{BPM}$ and maximum value of 100 BPM. Based on the abovementioned criteria, we programmed the mobile node to set off an event alarm if it received a value above 100 BPM or 60 BPM, respectively.

Subjects participating in the study were asked to totally relax in order to lower their BPM, establish baselines and to try to activate the alarm. The subjects registered no readings below 60 BMP under normal relaxed circumstances. Unlike, we were able to validate the 100 BPM criteria by having each subject exercise on an elliptical treadmill for 10, 3-minute periods. This permitted the subjects' heart rates to surpass the $100 \mathrm{BPM}$ value, which was consistently achieves as was predicted. Importantly, each time the subjects' heart rates exceeded the 100 BPM limit, the events were transmitted to the network and received by the SINK. The data were then uploaded by the web application to the interface shown in Figure 11.

Figure 11. Web System Alert Interface.

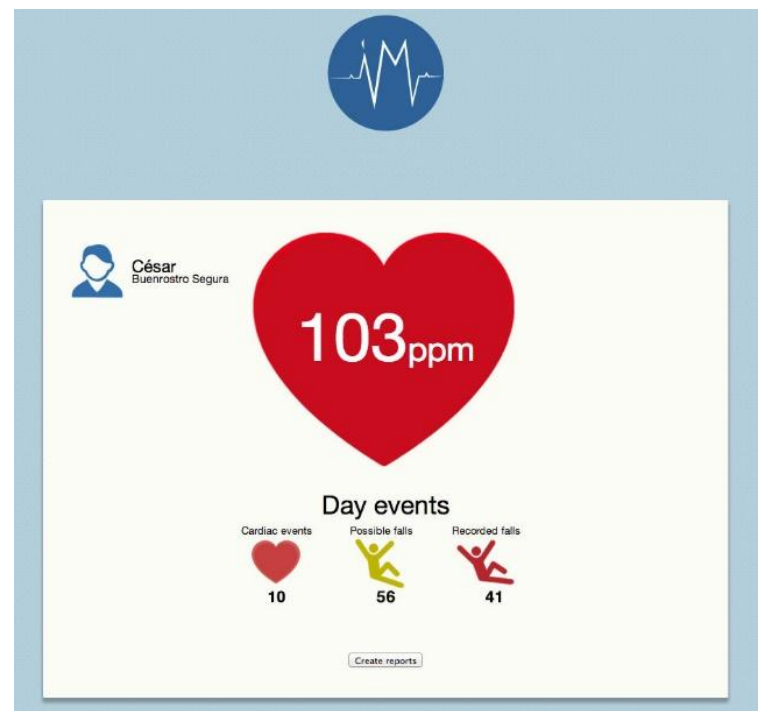

All heart rate event alerts reach the mobile by means of push notifications. These push notifications are then handled by the software application to be visualized. Figure 12 shows the alert notification as viewed on a mobile device.

Figure 12. Push notification generated for the heart rate event.

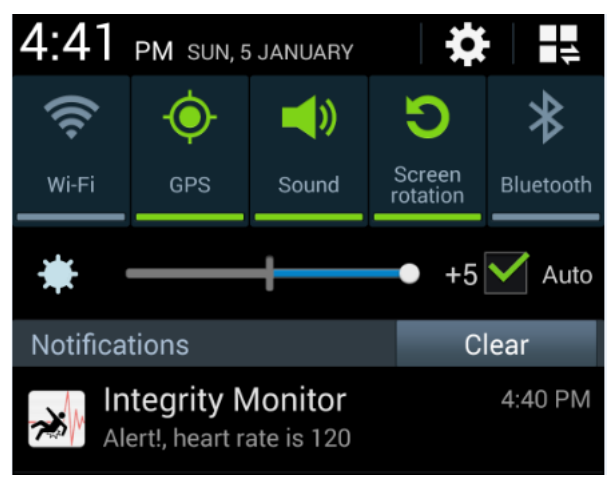


The results reported in Sections 5.1 and 5.2 involving patient monitoring were carried out under the supervision of the head of the geriatric department of the Institute of Safety and Social Services for State Workers (ISSSTE) of the state of Colima, Mexico.

\subsection{Network Results}

As far as the network is concerned, both the mobile device and the WSN employ a 128-bit AES encrypting mechanism. Two tests were performed to validate the safety device. Two tests were performed to validate the safety device. The first test consisted in introducing an unknown node from an outside network, which in contrast with network nodes, does not have its AES encrypting mechanism enabled. The second test consisted in introducing another unknown node that had an AES key enabled, but which did not correspond to the one used by the WSN. In both cases, the external nodes try to capture HELLO packets transmitted within the WSN. Table 4 shows the structure of packets collected by a network node within the WSN. Each packet begins with an identical byte of information. This is then followed by two bytes to indicate the packet length. The next byte defines the frame type which corresponds to the IEEE 802.15.4 standard $(0 \times 81-\mathrm{R} \times 16$ indicator $)$, followed by 2 bytes for the source address, 1 byte for the RSSI, and 1 byte for the method sent $(0 \times 00$ for unicast, $0 \times 02$ for broadcast), which, in this case, is broadcast mode. Finally, each packet contains a 2-byte payload and a 1-byte checksum to verify the integrity of the packet.

Table 4. HELLO Packet Structure.

\begin{tabular}{|c|c|c|c|c|c|c|c|}
\hline \multicolumn{8}{|c|}{ HELLO Packet Structure } \\
\hline $\begin{array}{l}\text { Begin } \\
\text { (1byte) }\end{array}$ & $\begin{array}{l}\text { Length } \\
\text { (2bytes) }\end{array}$ & $\begin{array}{c}\text { API } \\
\text { (1byte) }\end{array}$ & $\begin{array}{l}\text { Src. Address } \\
\text { (2bytes) }\end{array}$ & $\begin{array}{c}\text { RSSI } \\
\text { (1byte) }\end{array}$ & Opt. (1byte) & $\begin{array}{l}\text { Payload } \\
\text { ( } 2 \text { bytes })\end{array}$ & CS (1byte) \\
\hline $0 \times 7 \mathrm{E}$ & $0 \times 00 \quad 0 \times 07$ & $0 \times 81$ & $0 \times 00 \quad 0 \times 15$ & $0 \times 24$ & $0 \times 02$ & $0 \times 48 \quad 0 \times 01$ & $0 \times \mathrm{FF}$ \\
\hline
\end{tabular}

Figure 13a provides the results of the first security test. Results show that HELLO packets generated by a network node (left terminal) cannot be intercepted (right terminal) because the unknown node does not share the same AES mechanism. Figure 13b provides results that show that an unknown node can receive HELLO packets, but cannot decipher the 11-byte packets as originally sent because they do not possess the same key. An analysis of the packet received by the right terminal external device shows that the first byte is a packet start character $(0 \times 7 \mathrm{E})$. The following two bytes provide the packet length $(0 \times 000 \mathrm{~F})$, which in this case is 15 bytes, excluding the start bytes, length and checksum. The next byte indicates the frame type, which corresponds to the IEEE 802.15.4 standard $4(0 \times 80)$ that defines the MAC address used $(64$ bits $)$. For this reason, the next 8 bytes of the frame contain the physical address of the source node $(0 \times 0013$ A20040905DA6). The next byte of the frame indicates the packet's RSSI $(0 \times 24)$. The subsequent byte $(0 \times 02)$ whether the packet was transmitted by unicast or broadcast, which in this case was broadcast. The following 4 bytes $(0 \times$ A6FB643C) provide the packet payload. Lastly, the final byte provides the checksum $(0 \times 90)$. A comparison of the original data with the information acquired by the unknown node shows that it could properly get the starting character of the packet, its RSSI, the broadcast mode and the checksum. However, it could not obtain packet information corresponding to the length, frame type, source address and payload correctly. The unknown node cannot acquire this information because it does not 
possess the network encryption key. Experimental results show, in sum, that critical information regarding network information cannot be successfully obtained by a node that does not form part of the network.

Figure 13. (a) Security Test 1; (b) Security Test 2.
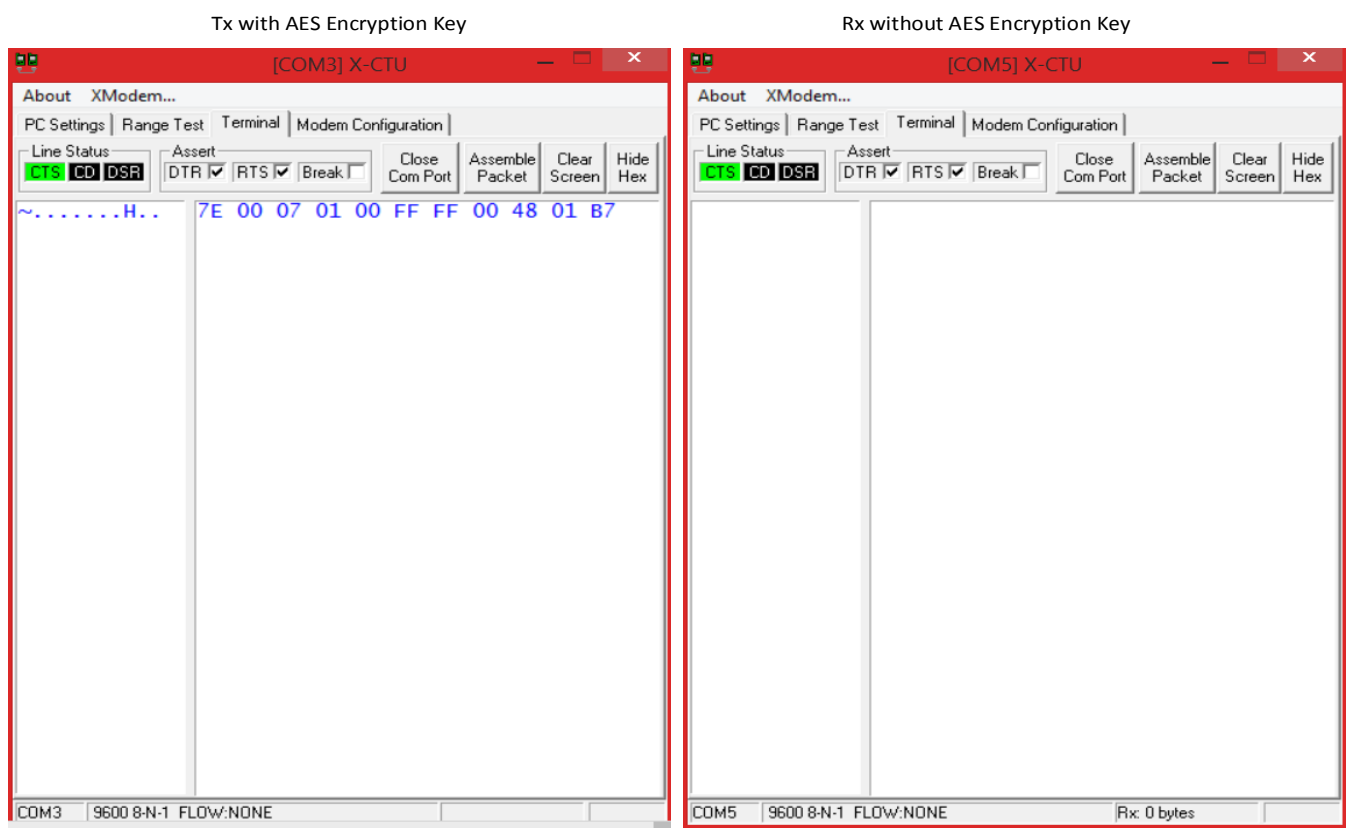

(a)
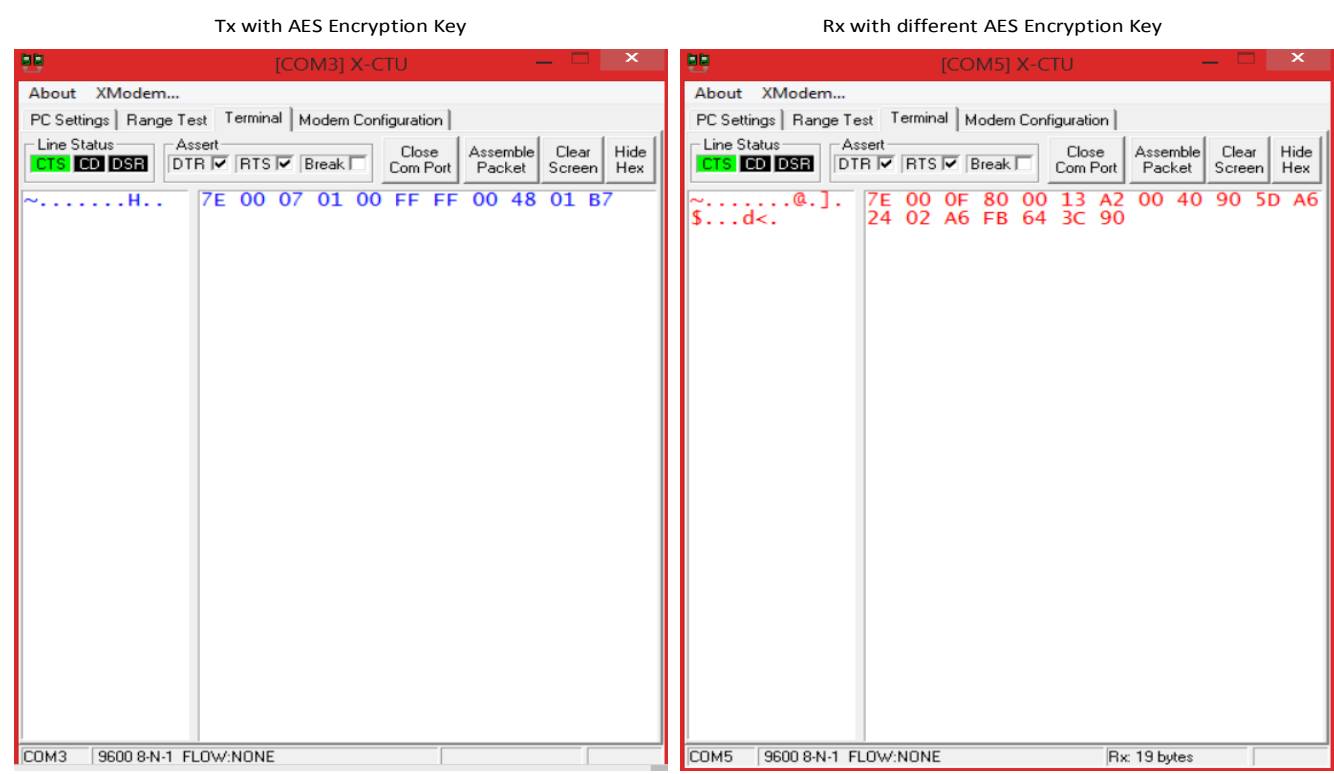

(b)

Once we tested the security of the WSN network, we proceeded to analyze and validate the functioning of the network. As mentioned previously, five infrastructure nodes were installed in the home to form a network which could communicate remotely to a mobile node, employing the routing protocol our team developed. Figure 14 illustrates the network configuration employed in our proposed network architecture, which employs two Cluster Head devices (ID16 and ID 64), two End Devices (ID 24 and ID 40), a mobile node (ID 32) and a SINK (ID 8). 
Figure 14. Created network.

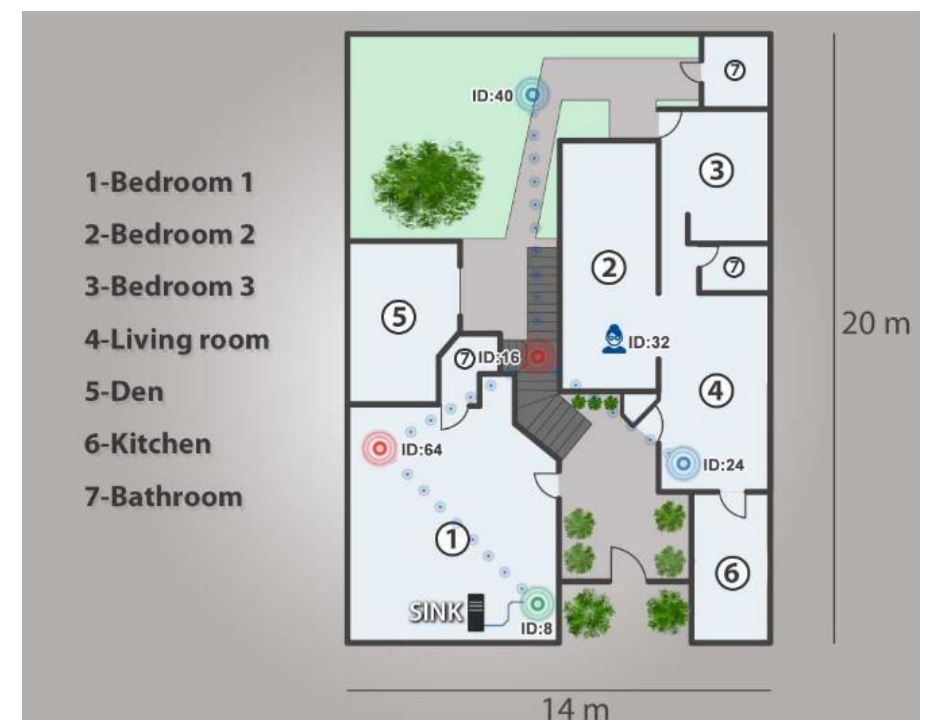

Once the network is formed, communication between the mobile node and the infrastructure can begin and data can be sent. In this test, a person representing a mobile node moved freely throughout the home to determine the number of hops and RSSI. To do this, data packets were generated and routed to the SINK, as shown in Table 5.

Table 5. Scenario routing table.

\begin{tabular}{cccc}
\hline ID Node & Trunk Destination & Hops & Link RSSI \\
\hline 8 & D/A & D/A & D/A \\
16 & 64 & 2 & $-64 \mathrm{dBm}$ \\
24 & 16 & 3 & $-57 \mathrm{dBm}$ \\
32 & Dynamic & Dynamic & D/A \\
40 & 16 & 3 & $-47 \mathrm{dBm}$ \\
64 & 8 & 1 & $-53 \mathrm{dBm}$ \\
\hline
\end{tabular}

A total of 50 events (40 different falls and 10 tachycardia events) were simulated under test conditions using healthy subjects. Each even generated packets that possessed a sequence number and an event identifier. Adding these values to the DATA packets allowed us to identifying the totality number of packets that were generated and received. From this data set, it was possible to determent the percentage of lost packets and the number of packets corresponding to each event. Specific information is provided by Table 6 .

Table 6. Received DATA Packets.

\begin{tabular}{ccccc}
\hline $\begin{array}{c}\text { Total } \\
\text { Packets }\end{array}$ & $\begin{array}{c}\text { Fall DATA } \\
\text { Packets }\end{array}$ & $\begin{array}{c}\text { Heart Rate DATA } \\
\text { Packets }\end{array}$ & Lost Packets & $\begin{array}{c}\text { Percent of DATA } \\
\text { Lost Packets }\end{array}$ \\
\hline 109 & 97 & 10 & 2 & $1.83 \%$ \\
\hline
\end{tabular}

According to the information provided by Table 6, of the 109 DATA packets received, 97 were attributable to fall events, 10 to heart rate events and only two packets were lost, which represents a $1.83 \%$ rate of error. 


\section{Conclusions and Future Work}

This paper has presented a Home Care Monitoring System (WiSPH), which represents a potentially valuable tool to assist caretakers, family members or health care practitioners to monitor the heart rate and potentially dangerous falls of elderly patients who can still live independent, but assisted lifestyles. The proof of concept testing presented in this work permits us to conclude:

- WiSPH implements a security mechanism (AES) that does not allow nodes from outside the network to decipher data.

- The routing algorithm, based on the IEEE 802.15.4 standard, functions efficiently in health applications as only two of the 109 DATA packets that were generated in this study were lost, representing less than a $1.8 \%$ of the packets sent.

- An efficient algorithm was developed to detect falls which correctly reported $100 \%$ of serious falls.

- The already established value for tachycardia made it possible to efficiently identify the 10 events that were programmed in this test, resulting in a $100 \%$ success rate. In contrast, the value for a low heart rate (bradycardia) could not be validated because subjects could not lower their heart rates below the 70 BPM criteria.

- The monitoring function of the network and alert interface worked well in conjunction. Of a total of 109 push notifications, caretakers $100 \%$ of the real-time alerts.

In sum, WiSPH employs important features from various platforms/systems that have been previously compared in this work. WiSPH implements an effective security mechanism and has relatively low power consumption. Our system combines these two characteristics with an efficient fall detection algorithm and an accurate means to detect established thresholds for bradycardia and tachycardia. WiSPH also possesses the flexibility and scalability to add other sensors because the routing algorithm provides the necessary QoS. The abovementioned characteristics not only permit WiSPH to be used by geriatric patients living at home, but with a wide variety of health care scenarios where mobile monitoring of different physiological variables is necessary. In conclusion, the variety of parameters WiSPH effectively monitors and reports on favorably compares to systems that are presently available in the marketplace.

Future work will include in-depth simulations to validate the system performance, in particular to simulate multiple nodes in different spatial areas to confirm its scalability. These simulations will provide the antecedents before performing large-scale real-world implementation inside a regional hospital. Additionally, subsequent work will include optimizing the PNR algorithm to detect different the severity of falls by means of combining enhanced accelerometer data with the accompanying heart rate changes. Another area of work in the future is to optimize the system to include greater personalization according to a patient's specific physiological variables, including amount of nutrition, age, weight, body fat, triglycerides, cholesterol etc. Other parameters can include stress test results to set a baseline for elderly patients that can be introduced into the system. 


\section{Acknowledgments}

The authors wish to thank all the partners involved with the development of the system, especially to Jorge Landín Delgado for his perspective on the provision of remote health care and Siteldi Solutions (www.siteldisolutions.mx) and financial support from CONACYT, project number 209240.

\section{Author Contributions}

Pedro Magaña Espinoza designed the experiments, and drafted the manuscript. Raúl Aquino Santos provided useful suggestions and edited the draft. Néstor Cárdenas Benítez deployed the WSN Infrastructure. Jose Aguilar Velasco programmed the SINK. César Buenrostro Segura programmed the mobile application. Arthur Edwards Block provided English grammar corrections and edited the draft. Aldo Medina Cass provided English grammar suggestions.

\section{Conflicts of Interest}

The authors declare no conflict of interest.

\section{References}

1. WHO Website. 10 Facts on Ageing and the Life Course. Available online: http://www.who.int/ features/factfiles/ageing/es/index.html (accessed on 23 December 2013).

2. WHO Website. Interesting Facts about Ageing. Available online: http://www.who.int/ ageing/about/facts/en/index.html (accessed on 23 December 2013).

3. WHO Website. Falls. Available online: http://www.who.int/mediacentre/factsheets/fs344/ en/index.html (accessed on 28 December 2013).

4. Boonyarattaphan, A.; Yan, B.; Sam, C. A security framework for e-health service authentication and e-health data transmission. In Proceedings of the 9th International Symposium on Communications and Information Technology (ISCIT 2009), Incheon, Korea, 28-30 September 2009; pp. 1213-1218.

5. Tan, O.; Wei Kiat, K.; Jamie, N.; Wong, A.; Tay, Z.; Helander, M.G. Are working adults ready to accept e-health at home? In Proceedings of the 11th International Conference on e-Health Networking, Applications and Services (Healthcom 2009), Sidney, Australia, 16-18 December 2009; pp. 54-59.

6. Alemdar, H.; Ersoy, C. Wireless sensor networks for healthcare: A survey. Comput. Netw. 2010, $54,2688-2710$.

7. Lu, C.-H.; Fu, L.-C. Robust location-aware activity recognition using wireless sensor network in an attentive home. IEEE Trans. Autom. Sci. Eng. 2009, 6, 598-609.

8. Philipose, M.; Consolvo, S.; Fishkin, K.; Smith, P.I. Fast, detailed inference of diverse daily human activities. In Proceedings of the Sixth International Conference on Ubiquitous Computing, Nottingham, UK, 7-10 September 2004. 
9. Tabar, A.M.; Keshavarz, A.; Aghajan, H. Smart home care network using sensor fusion and distributed vision-based reasoning. In Proceedings of the 4th ACM International Workshop on Video Surveillance and Sensor Networks, Santa Barbara, CA, USA, 27 October 2006; ACM: Santa Barbara, CA, USA, 2006; pp. 145-154.

10. Wang, C.-C.; Chiang, C.-Y.; Lin, P.-Y.; Chou, Y.-C.; Kuo, I.T.; Huang, C.-N.; Chan, C.-T. Development of a fall detecting system for the elderly residents. In Proceedings of the 2nd International Conference on Bioinformatics and Biomedical Engineering (ICBBE 2008), Shanghai, China, 16-18 May 2008; pp. 1359-1362.

11. Yan, H.; Xu, Y.; Gidlund, M.; Nohr, R. An experimental study on home-wireless passive positioning. In Proceedings of the Second International Conference on Sensor Technologies and Applications (SENSORCOMM'08), Cap Esterel, France, 25-31 August 2008; pp. 223-228.

12. Marco, A.; Casas, R.; Falco, J.; Gracia, H.; Artigas, J.I.; Roy, A. Location-based services for elderly and disabled people. Comput. Commun. 2008, 31, 1055-1066.

13. Lopez-Nores, M.; Pazos-arias, J.; Garcia-Duque, J.; Blanco-Fernandez, Y. Monitoring medicine intake in the networked home: The icabinet solution. In Proceedings of the Second International Conference on Pervasive Computing Technologies for Healthcare (PervasiveHealth 2008), Tampere, Finland, 30 January-1 February 2008; pp. 116-117.

14. Pang, Z.; Chen, Q.; Zheng, L. A pervasive and preventive healthcare solution for medication noncompliance and daily monitoring. In Proceedings of the 2nd International Symposium on Applied Sciences in Biomedical and Communication Technologies (ISABEL 2009), Bratislava, Slovakia, 24-27 November 2009; pp. 1-6.

15. Wood, A.; Stankovic, J.A.; Virone, G.; Selavo, L.; He, Z.; Cao, Q.; Thao, D.; Wu, Y.; Fang, L.; Stoleru, R. Context-aware wireless sensor networks for assisted living and residential monitoring. IEEE Netw. 2008, 22, 26-33.

16. Baker, C.R.; Armijo, K.; Belka, S.; Benhabib, M.; Bhargava, V.; Burkhart, N.; Der Minassians, A.; Dervisoglu, G.; Gutnik, L.; Haick, M.B.; et al. Wireless sensor networks for home health care. In Proceedings of the 21st International Conference on Advanced Information Networking and Applications Workshops (AINAW'07), Ontario, ON, Canada, 21-23 May 2007; pp. 832-837.

17. Jiménez, V.P.G.; Armada, A.G. Field measurements and guidelines for the application of wireless sensor networks to the environment and security. Sensors 2009, 9, 10309-10325.

18. Egbogah, E.E.; Fapojuwo, A.O. A survey of system architecture requirements for health care-based wireless sensor networks. Sensors 2011, 11, 4875-4898.

19. González, A.; Aquino, R.; Mata, W.; Ochoa, A.; Saldaña, P.; Edwards, A. Open-wise: A solar powered wireless sensor network platform. Sensors 2012, 12, 8204-8217.

20. Malan, D. Codeblue: An ad hoc sensor network infrastructure for emergency medical care. In Proceedings of the MobiSys 2004 Workshop on Applications of Mobile Embedded Systems (WAMES 2004), Boston, MA, USA, 6 June 2004.

21. Ko, J.; Lim, J.H.; Chen, Y.; Rvăzvan Musvaloiu-E, Z.; Terzis, A.; Masson, G.M.; Gao, T.; Destler, W.; Selavo, L.; Dutton, R.P. Medisn: Medical emergency detection in sensor networks. ACM Trans. Embed. Comput. Syst. 2010, 10, 1-29.

22. Hu, F.; Jiang, M.; Celentano, L.; Xiao, Y. Robust medical ad hoc sensor networks (MASN) with wavelet-based ECG data mining. Ad Hoc Netw. 2008, 6, 986-1012. 
23. Mitra, U.; Emken, B.A.; Sangwon, L.; Ming, L.; Rozgic, V.; Thatte, G.; Vathsangam, H.; Zois, D.; Annavaram, M.; Narayanan, S.; et al. Knowme: A case study in wireless body area sensor network design. IEEE Commun. Mag. 2012, 50, 116-125.

24. Custodio, V.; Herrera, F.; López, G.; Moreno, J. A review on architectures and communications technologies for wearable health-monitoring systems. Sensors 2012, 12, 13907-13946.

25. Tarng, W.; Lin, C.-H.; Liou, H.-H. Applications do wireless sensor networks in fall detection for senior people. Int. J. Comput. Sci. Inf. Technol. 2012, 4, 79-95.

26. Karantonis, D.M.; Narayanan, M.R.; Mathie, M.; Lovell, N.H.; Celler, B.G. Implementation of a real-time human movement classifier using a triaxial accelerometer for ambulatory monitoring. IEEE Trans. Inf. Technol. Biomed. 2006, 10, 156-167.

27. Agruss, N.S.; Rosin, E.Y.; Adolph, R.J.; Fowler, N.O. Significance of chronic sinus bradycardia in elderly people. Circulation 1972, 46, 924-930.

28. Budzikowski, A.; Cho, C. Atrial Tachycardia. Available online: http://emedicine.medscape.com/ article/151456-overview (accessed on 4 March 2014).

29. Kumar, P.; Lee, H.-J. Security issues in healthcare applications using wireless medical sensor networks: A survey. Sensors 2011, 12, 55-91.

30. Raspberry Pi. Available online: http://www.raspberrypi.org/ (accessed on 3 March 2014).

(C) 2014 by the authors; licensee MDPI, Basel, Switzerland. This article is an open access article distributed under the terms and conditions of the Creative Commons Attribution license (http://creativecommons.org/licenses/by/3.0/). 